\title{
The response of thrombosis in the portal vein or hepatic vein in hepatocellular carcinoma to radiation therapy
}

\author{
Bong Kyung Bae, MD, Jae-Chul Kim, MD \\ Department of Radiation Oncology, Kyungpook National University School of Medicine, Daegu, Korea
}

\begin{abstract}
Purpose: The purpose of current study is to evaluate the response of the patients with portal vein thrombosis (PVT) or hepatic vein thrombosis (HVT) in hepatocellular carcinoma (HCC) treated with three-dimensional conformal radiation therapy (3D-CRT). In addition, survival of patients and potential prognostic factors of the survival was evaluated.

Materials and Methods: Forty-seven patients with PVT or HVT in HCC, referred to our department for radiotherapy, were retrospectively reviewed. For 3D-CRT plans, a gross tumor volume (GTV) was defined as a hypodense filling defect area in the portal vein (PV) or hepatic vein (HV). Survival of patients, and response to radiation therapy (RT) were analyzed. Potential prognostic factors for survival and response to RT were evaluated.

Results: The median survival time of 47 patients was 8 months, with 1-year survival rate of 15\% and response rate of $40 \%$. Changes in Child-Pugh score, response to RT, Eastern cooperative oncology group performance status (ECOG PS), hepatitis C antibody (HCVAb) positivity, and additional post RT treatment were statistically significant prognostic factors for survival in univariate analysis ( $p=0.000, p=0.018, p=0.000, p=0.013$, and $p=0.047$, respectively). Of these factors, changes in Child-Pugh score, and response to RT were significant for patients' prognosis in multivariate analysis ( $p=0.001$ and $p=0.035$, respectively).

Conclusion: RT could constitute a reasonable treatment option for patients with PVT or HVT in HCC with acceptable toxicity. Changes in Child-Pugh score, and response to RT were statistically significant factors of survival of patients.
\end{abstract}

Keywords: Hepatocellular carcinoma, Portal vein, Hepatic vein, Radiotherapy

\section{Introduction}

Primary cancers of the liver represent the fifth most common malignancy worldwide and the second most common cause of death from cancer [1]. Surgical resection and orthotropic liver transplantation are the gold standard therapy, but this treatment option is limited to localized hepatocellular carcinoma (HCC) patients. Most patients have locally advanced disease at the time of diagnosis and are only candidates for palliative treatment.
For advanced HCC patients, more than $40 \%$ of patients have portal vein thrombosis (PVT), or hepatic vein thrombosis (HVT) [2-4]. These thrombi can result in intrahepatic metastasis, reduced hepatic blood stream, portal hypertension, ascites, variceal rupture, and hepatic dysfunction [5]. Transarterial chemoembolization (TACE) delivers chemotherapeutic agents to HCC via the hepatic artery and releases the loaded drugs to a local area, with the embolic effect leading to tumor ischemia. If the blood supply of the portal vein is not patent, the blockage of hepatic artery can have catastrophic

Received 6 February 2016, Revised 23 May 2016, Accepted 21 June 2016.

Correspondence: Jae-Chul Kim, MD, Department of Radiation Oncology, Kyungpook National University School of Medicine, 680 Gukchaebosang-ro, Jung-gu, Daegu 41944, Korea. Tel: +82-53-200-5352, Fax: +82-53-426-3303, E-mail: jckim@knu.ac.kr

(C) This is an Open Access article distributed under the terms of the Creative Commons Attribution Non-Commercial License (http://creativecommons.org/ licenses/by-nc/4.0/) which permits unrestricted non-commercial use, distribution, and reproduction in any medium, provided the original work is properly cited.

www.e-roj.org 
consequences. Thus, if the blood flow of the portal vein is obstructed by a thrombus, any additional treatment of HCC with TACE is considered risky, and has yielded unsatisfactory results [6-9]. For these reasons, prognosis of HCC patients with PVT or HVT is known to be poor. Without treatment, the survival window for these patients is under 3 months $[9,10]$.

Historically, radiation therapy (RT) for liver tumor was limited because liver was known to be a radiosensitive organ. Yet with advances in imaging, treatment planning, and treatment delivery, now we could make an attempt to deliver tumoricidal doses to target areas without incurring significant side effects [5,11-14]. Partial liver irradiation has shown some promising results in patients with unresectable HCC; promising outcomes were also observed in patients with PVT who were treated with RT $[9,15-18]$. The primary end point of this study was to evaluate the response of PVT or HVT treated with RT for the evaluation of the efficacy and feasibility of RT. Secondary end points of this study were to evaluate the survival of patients, and to evaluate the potential prognostic factors that might affect the patients' survival. In addition, early toxicities and late toxicities, especially to liver, were reviewed.

\section{Materials and Methods}

\section{Patients and radiotherapy planning}

From August 2007 to January 2015, a total of 64 patients were treated with RT for PVT or HVT in HCC. Patients were clinically diagnosed as HCC based on American Association for the Study of Liver Disease guideline [19]. Tumor thrombus was diagnosed based on the intraluminal filling defect lesion found on contrast-enhanced computed tomography (CT) [5]. Of these patients, patients with Child-Pugh classification $C_{\text {, or }}$ with Eastern Cooperative Oncology Group (ECOG) performance status of 3 or 4 , or who lacked of follow-up studies for evaluation of response, or who did not completed planned radiotherapy treatment were excluded. Forty-seven patients were included in the current study after exclusion, and the characteristics of the patients are listed in Table 1.

All patients underwent enhanced abdominal radiotherapy planning-CT (RTP-CT) for planning of radiotherapy in the supine position, with both arms raised above the head. Patients took RTP-CT under free breathing condition, and the slice thickness of RTP-CT was $5 \mathrm{~mm}$. Normal liver, spinal cord, bilateral kidneys, and stomach were delineated as organs at risk (OAR). Gross tumor volume (GTV) was defined as a hypodense filling defect of the portal or hepatic vein. If the distal end of filling defect lesion was unclear, we included up to segmental branches of portal vein (PV). And if primary tumor was close to the thrombus, included of primary tumor in to GTV was considered. A planning target volume (PTV) of a 1-2 cm margin from the GTV was given to include daily set-up variation and respiratory movement.

RT was delivered using four-field coplanar beams using 6 or $10 \mathrm{MV}$ photons under free breathing condition. In some cases, noncoplanar beams were used to avoid normal tissue overdose, especially to kidneys, and to improve the PTV dose coverage.

Two treatment schedules, $40 \mathrm{~Gy}$ in 16 fractions (fraction size, $2.5 \mathrm{~Gy}$ ) or $45 \mathrm{~Gy}$ in 25 fractions (fraction size, $1.8 \mathrm{~Gy}$ ), were administered. If a thrombus was large or located close to normal organs such as the small bowel, the treatment schedule of $45 \mathrm{~Gy}$ in 25 fractions was prescribed. If a thrombus was small or if the patient performance was poor, the schedule of $40 \mathrm{~Gy}$ in 16 fractions was prescribed. Both treatment options were translated into biologically effective doses (BEDs) of 53.1 $\mathrm{Gy}_{10}$ and $50 \mathrm{~Gy}_{10}$, respectively, as the tumor $\alpha / \beta$ ratio of 10 .

For the planning of RT, the following normal organ dose constraints were adopted. The volume of the normal liver receiving more than $25 \mathrm{~Gy}\left(\mathrm{~V}_{25}\right)$ was kept below 50\%. The mean dose to whole liver was kept below $30 \mathrm{~Gy}$. The maximal dose to the spinal cord was kept below $45 \mathrm{~Gy}$. The mean dose to both kidneys was kept below $20 \mathrm{~Gy}$.

\section{Evaluation and statistical analysis}

World Health Organization criteria, or Response Evaluation Criteria in Solid Tumors (RECIST), or modified RECIST is usually used for the assessment of treatment response. But as tumor thrombus is considered as nonmeasurable lesion, we could not use the methods described above, and had to use our own criteria. Responses to RT were evaluated by inspecting 3-phase CT images and comparing the thrombus size in the CT taken before RT with the thrombus size in the CT taken 2-3 months after RT. When no CT images were available 2-3 months after $R T$, a shorter time interval between the RT and evaluation was accepted. Complete response (CR) was defined as the complete disappearance of PVT or HVT; partial response (PR), was defined as greater than 30\% reduction in length of major axis of thrombus in the axial CT slices; and progressive disease (PD), as a more than $20 \%$ increase in length of major axis of the PVT or HVT. Stable disease (SD) was defined as an intermediate state between PR and PD. The response rate was defined as a sum of CR and PR (Fig. 1). Progression-free time was evaluated by comparing the size of thrombus in the follow-up CT scans. If the size of the thrombus starts to increase compared to 

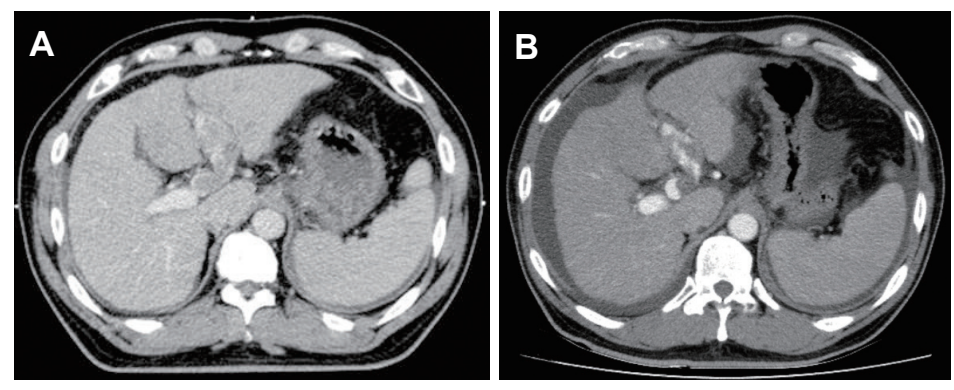

Fig. 1. The case of tumor response evaluation in a 47-year old man. The patient was diagnosed with hepatocellular carcinoma and referred to our department for radiotherapy of thrombus in the left portal vein. (A) The initial radiation therapy planning-computed tomography, showing the thrombus in the left portal vein and no portal flow. (B) The follow-up abdominal computed tomography, taken 24 days after completion of radiotherapy. The thrombus is reduced in size, and the portal flow is renewed.

the previous imaging study, we defined it as progression. And the time between start of RT and progression was defined as progression-free time.

The overall survival rate was calculated from the start of $\mathrm{RT}$ to the date of death or to the latest follow-up. Univariate analysis was performed by log-rank test for evaluating potential prognostic factors that could be important for the patients' survival. Factors that reached statistical significance ( $p<0.05)$, or closely approached statistical significance $(p<$ 0.10 ) based on the univariate analysis were included in the multivariate analysis by using the Cox regression model.

\section{Toxicities}

Acute toxicity during or after radiotherapy was evaluated according to the Common Terminology Criteria for Adverse Events (CTCAE), ver. 4.03. For the evaluation of late injury, all patients were examined for exhibiting any signs or symptoms of radiation-induced liver disease (RILD) after RT. RILD in the current study was defined by Lawrence's criterion. The classic RILD was defined as a RILD that occurred within 4 months after completing RT, with an elevation of the alkaline phosphatase (ALP) level to at least twice the upper normal level or nonmalignant ascites. The non-classic RILD was defined as an elevation of the enzymes aspartate transaminase (AST) or alanine transaminase (ALT) at least fivefold above the upper limit or the pretreatment level [20-23]. Change of ChildPugh score after RT was also evaluated. If there was change in Child-Pugh score of 2 or more points, it was considered as development of complication [24].

\section{Results}

There were 37 male and 10 female patients with the median age of 60 years (range, 38 to 79 years). ECOG performance status was 0 in 6 patients, 1 in 33 patients, and 2 in 8 patients. Child-Pugh classification was A in 31 patients, and B in 16 patients. Thirty-three patients were positive for hepatitis $B$
Table 1. Characteristics of 47 patients with the portal vein thrombi or hepatic vein thrombi in hepatocellular carcinoma

\begin{tabular}{|c|c|}
\hline Characteristic & No. $(\%)$ \\
\hline \multicolumn{2}{|l|}{ Gender } \\
\hline Male & 37 (79) \\
\hline Female & $10(21)$ \\
\hline \multicolumn{2}{|l|}{ Age (yr) } \\
\hline$<60$ & $24(51)$ \\
\hline$\geq 60$ & $23(49)$ \\
\hline \multicolumn{2}{|l|}{ ECOG performance status } \\
\hline 0 & $6(13)$ \\
\hline 1 & $33(70)$ \\
\hline 2 & $8(17)$ \\
\hline \multicolumn{2}{|l|}{ Child-Pugh classification } \\
\hline$A$ & $31(66)$ \\
\hline$B$ & $16(34)$ \\
\hline \multicolumn{2}{|l|}{ Involving site } \\
\hline Main trunk & $27(57)$ \\
\hline PV branch & $10(21)$ \\
\hline HV & $5(11)$ \\
\hline $\mathrm{PV}$ and $\mathrm{HV}$ & $5(11)$ \\
\hline \multicolumn{2}{|l|}{$\mathrm{HBsAg}$} \\
\hline Positive & $33(70)$ \\
\hline Negative & $14(30)$ \\
\hline \multicolumn{2}{|l|}{$\mathrm{HBeAg}$} \\
\hline Positive & $8(17)$ \\
\hline Negative & $35(74)$ \\
\hline Data missing & $4(9)$ \\
\hline \multicolumn{2}{|l|}{ HCVAb } \\
\hline Positive & $4(9)$ \\
\hline Negative & $39(82)$ \\
\hline Data missing & $4(9)$ \\
\hline \multicolumn{2}{|c|}{ Previous treatment before radiotherapy } \\
\hline$(+)$ & $35(74)$ \\
\hline$(-)$ & $12(26)$ \\
\hline \multicolumn{2}{|c|}{ Additional treatment after radiotherapy } \\
\hline$(+)$ & $9(19)$ \\
\hline$(-)$ & $38(81)$ \\
\hline
\end{tabular}

ECOG, Eastern Cooperative Oncology Group; PV, portal vein; HV, hepatic vein; $\mathrm{HBsAg}$, hepatitis B surface antigen; $H B e A g$, hepatitis $B$ envelope antigen; HCVAb, hepatitis $C$ antibody. 
Table 2. Univariate analysis of potential prognostic factors

\begin{tabular}{|c|c|c|c|}
\hline Factor & No. & Median survival \pm SE & $p$-value \\
\hline Gender & & & 0.373 \\
\hline Male & 37 & $7.0 \pm 1.14$ & \\
\hline Female & 10 & $11.2 \pm 4.87$ & \\
\hline Age (yr) & & & 0.094 \\
\hline$\leq 60$ & 27 & $6.6 \pm 1.35$ & \\
\hline$>60$ & 20 & $12.7 \pm 3.47$ & \\
\hline Thrombus location & & & 0.371 \\
\hline Main trunk & 27 & $9.5 \pm 1.34$ & \\
\hline PV branch & 10 & $3.7 \pm 2.96$ & \\
\hline $\mathrm{HV}$ & 5 & $7.0 \pm 2.39$ & \\
\hline Both PV and HV & 5 & $4.0 \pm 0.85$ & \\
\hline Level of thrombus & & & 0.225 \\
\hline Main trunk or IVC & 34 & $9.1 \pm 2.00$ & \\
\hline PV branch or HV & 13 & $7.0 \pm 3.34$ & \\
\hline GTV volume (mL) & & & 0.498 \\
\hline$\leq 50$ & 20 & $9.5 \pm 2.44$ & \\
\hline$>50$ & 27 & $7.0 \pm 1.86$ & \\
\hline Albumin (g/dL) & & & 0.297 \\
\hline$\leq 3.5$ & 25 & $9.1 \pm 1.57$ & \\
\hline$>3.5$ & 22 & $6.6 \pm 1.70$ & \\
\hline Child-Pugh classification & & & 0.257 \\
\hline A & 31 & $10.7 \pm 1.15$ & \\
\hline B & 16 & $6.1 \pm 0.89$ & \\
\hline Child-Pugh score change & & & 0.000 \\
\hline Positive & 31 & $4.2 \pm 0.40$ & \\
\hline Negative & 16 & $11.2 \pm 1.69$ & \\
\hline $\mathrm{HBsAg}$ & & & 0.714 \\
\hline Positive & 25 & $7.0 \pm 1.56$ & \\
\hline Negative & 22 & $9.5 \pm 2.91$ & \\
\hline $\mathrm{HBeAg}$ & & & 0.455 \\
\hline Positive & 8 & $6.6 \pm 1.32$ & \\
\hline Negative & 35 & $8.2 \pm 2.07$ & \\
\hline Data missing & 4 & - & \\
\hline $\mathrm{HCVAb}$ & & & 0.013 \\
\hline Positive & 4 & $3.6 \pm 0.95$ & \\
\hline Negative & 39 & $9.1 \pm 1.00$ & \\
\hline Data missing & 4 & - & \\
\hline Previous treatment before radiotherapy & & & 0.338 \\
\hline$(+)$ & 35 & $8.3 \pm 1.63$ & \\
\hline$(-)$ & 12 & $6.1 \pm 1.16$ & \\
\hline Additional treatment after radiotherapy & & & 0.047 \\
\hline$(+)$ & 9 & $12.7 \pm 2.35$ & \\
\hline$(-)$ & 38 & $6.6 \pm 1.19$ & \\
\hline ECOG performance status & & & 0.000 \\
\hline $0-1$ & 39 & $9.1 \pm 1.47$ & \\
\hline 2 & 8 & $2.6 \pm 0.30$ & \\
\hline RT schedule & & & 0.913 \\
\hline 40 Gy/16 fractions & 19 & $10.8 \pm 2.75$ & \\
\hline 45 Gy/25 fractions & 28 & $8.2 \pm 1.18$ & \\
\hline RT response & & & 0.018 \\
\hline Responder & 19 & $12.7 \pm 4.76$ & \\
\hline Non-responder & 28 & $7.0 \pm 1.79$ & \\
\hline
\end{tabular}

SE, standard error; PV, portal vein; HV, hepatic vein; IVC, inferior vena cava; GTV, gross tumor volume; HBsAg, hepatitis B surface antigen; $\mathrm{HBeAg}$, hepatitis B envelope antigen; HCVAb, hepatitis C antibody; ECOG, Eastern Cooperative Oncology Group; RT, radiation therapy. 


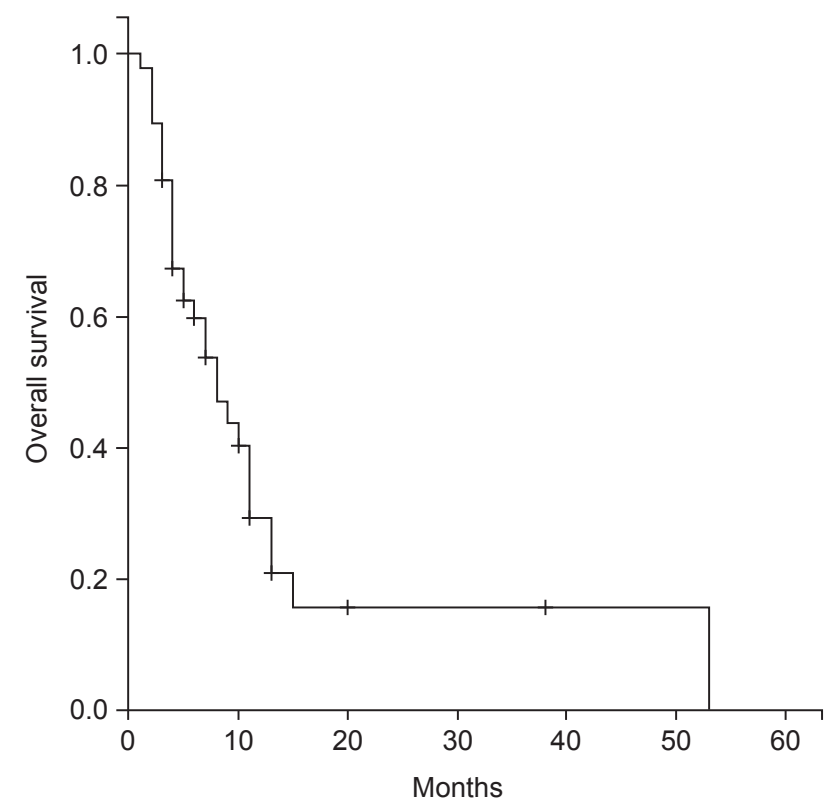

Fig. 2. The overall survival curve for the 47 patients treated with three-dimensional conformal radiation therapy (3D-CRT) for portal vein or hepatic vein thrombosis in hepatocellular carcinoma.

surface antigen ( $\mathrm{HBsAg}), 8$ patients were positive for hepatitis $B$ envelope antigen ( $\mathrm{HBeAg}$ ), and 4 patients were positive for hepatitis $\mathrm{C}$ antibody (HCVAb). Thirty-five patients had previous treatments including surgery, TACE, and/or radiofrequency ablation (RFA) before they were referred for radiotherapy. After RT, 9 patients had additional treatments, such as TACE. Further patient characteristics are listed in Table 1.

The median survival time of 47 patients was 8 months, and 1-year survival rate was 15\% (Fig. 2). The follow-up CT for evaluation of response was taken with the median of 68 days (range, 24-174 days) after completion of RT. After RT, no patient exhibited CR, 19 patients (40\%) exhibited PR, 24 patients (51\%) exhibited SD, and 4 patients (9\%) exhibited PD. The response rate $(C R+P R)$ was 40\% (19 patients). During follow-up after RT, 20 patients (43\%) showed progression of previously irradiated field. Median time to progression from start of RT was 127.5 days (range, 45-291 days).

Correlations between the responses to RT and GTVs were evaluated. The mean GTV for the patients who exhibited PR (19 patients) was $65.6 \mathrm{~mL}$, with the standard deviation of 36.1. The mean GTV for the patients who exhibited SD (24 patients) was $71.6 \mathrm{~mL}$, with the standard deviation of 68.9. The mean GTV for the patients who exhibited PD (4 patients) was $83.25 \mathrm{~mL}$, with the standard deviation of 43.7. Though there was a trend toward smaller GTV for better response numerically, it did not show statistical significance in one-way ANOVA ( $p=0.837)$.

According to the univariate analysis, change in Child-Pugh score, positivity for HCVAb, additional treatment after RT, ECOG $P S$, and response to RT were statistically significant prognostic factors determining patients' survival $(p=0.000, p=0.013, p$ $=0.047, p=0.000$, and $p=0.018$, respectively). Age $(p=0.094)$ was statistically marginal factor determining the patients survival (Table 2). In the multivariate analysis of statistically significant or marginal factors of survival in the univariate analysis, change in Child-Pugh score and response to RT were statistically significant factors determining the patients' survival ( $p=0.001$ and $p=0.035$, respectively) (Table 3 ).

The major early complications after RT were general weakness and nausea. Five patients (10\%) complained of having grade 1 general weakness, and 1 patient (2\%) complained of having grade 2 general weakness. Five patients (10\%) experienced grade 1 nausea, and 1 patient (2\%) experienced grade 2 nausea. Two patients (4\%) experienced grade 1 abdominal pain, and 1 patient (2\%) experienced grade 2 abdominal pain. Four patients (8\%) were hospitalized owing to exhibiting grade 3 hematemesis during, or shortly after RT. All patients with hematemesis underwent esophago-gastroduodenoscopy (EGD) for evaluation and management of bleeding. The reason for hematemesis was discovered to be variceal bleeding. There was no evidence of gastric or duodenal ulcer or bleeding.

For evaluation of late toxicity, RILD and changes in Child-

Table 3. Multivariate analysis of potential prognostic factors

\begin{tabular}{llll}
\multicolumn{1}{c}{ Factor } & HR & $95 \% \mathrm{Cl}$ & $\mathrm{p}$-value \\
\hline Change in Child-Pugh score (increase vs. no change) & 7.218 & $2.281-22.843$ & 0.001 \\
RT response (responder vs. non-responder) & 2.588 & $1.070-6.263$ & 0.035 \\
ECOG performance status (0-1 vs. 2) & 2.930 & $0.993-8.651$ & 0.052 \\
HCVAb (positive vs. negative) & 3.231 & $0.919-11.354$ & 0.067 \\
Additional treatment after RT (additional treatment vs. no treatment) & 1.563 & $0.538-4.545$ & 0.412 \\
Age ( $\leq 60$ vs. $>60)$ & 1.388 & $0.539-3.577$ & 0.497 \\
\hline
\end{tabular}

$\mathrm{HR}$, hazard ratio; $\mathrm{Cl}$, confidence interval; RT, radiation therapy; ECOG, Eastern Cooperative Oncology Group; HCVAb, hepatitis C antibody. 
Pugh score were evaluated. The review of laboratory studies for evaluation was performed at the median of 3.1 months (0.9 to 9 months) after RT. Only 1 patient met the criteria for the non-classic RILD. No other patients met the criteria for the classic or non-classic RILD. Pre-treatment Child-Pugh score was mean 6.1, and post-treatment Child-Pugh score was mean 7.9 , which showed increase of 1.8 points. Twenty-five patients (53\%) showed increase of 2 or more points in Child-Pugh score.

\section{Discussion and Conclusion}

Liver has dual blood supply, from the portal vein and from the hepatic artery. If both blood supply lines are patent, HCC patients can be treated with TACE, because even if the hepatic artery is blocked, hepatocytes receive nutrients and oxygen through the portal vein. But if the portal system is blocked by a thrombus, it is not possible to treat patients with TACE, because hepatocytes do not have any blood supplied from either the hepatic artery or the portal vein following the blockage of the hepatic artery. Thus, one of the main goals in RT of PVT or HVT in HCC is to prepare an additional treatment, such as TACE, by recanalizing the portal system. Such additional treatment by utilizing the recanalized portal system could lead to an improved overall survival. In this study, patients who received additional treatments demonstrated better overall survival compared with patients who received no additional treatments (12.7 months and 6.6 months, respectively, $p=0.047$ ). And $\mathrm{RT}$ responders demonstrated better overall survival, compared with RT non-responders (12.7 months and 7.0 months, respectively, $p=0.018$ ). This suggests that patients with good response to RT could be candidates for additional treatments, and additional treatments were related to a better overall survival.

Child-Pugh score is one of the most important prognostic factors for patient survival [25-27]. Some reports had suggested that the overall survival of HVT or PVT patients could be affected by Child-Pugh score. In the current study, there was a trend favoring better median survival rates with lower Child-Pugh class (10.7 months for class A, 6.1 months for class B), though statistically insignificant ( $p=0.257)$. Such low statistical power in our study could be due to the small sample size and imbalanced patient groups (31 patients in the group of class $A$, and 16 patients in the group of class B). Although pre-treatment Child-Pugh score did not show statistical significance in survival, change in Child-Pugh score showed to be statistically significant factor of survival in both univariate, and multivariate analysis $(p=0.000$ and $p=0.001$ respectively).

The optimal RT dose for PVT or HVT remains debated. In previous studies, the prescribed $\mathrm{RT}$ dose was in the range of $30-72 \mathrm{~Gy}$, and the response rate was in the range of 26\%-56\% [5,25-29]. Ishikura et al. [28] reported the results of treating PVT with 50 Gy with a 2 Gy fraction size, combined with TACE. In their study, the response rate was 50\%, the 1-year survival rate was $25 \%$, and the median survival time was 5.4 months. Toya et al. [27] reported the results of treating PVT with 17.5 to 50.4 Gy RT with a 1.8 to 4 Gy fraction size. They obtained the response rate of $45 \%$, the 1-year survival rate of $39 \%$, and the median survival time of 9.6 months.

Some studies reported response rates to prescribed BED $[5,11,18,27]$. Kim et al. [5] reported that the response of PVT was higher if the prescribed BED was higher than $64 \mathrm{~Gy}_{10}(50 \%$ vs. 0\%). Lee et al. [18] reported that the response of PVT was higher if the prescribed BED was higher than $60 \mathrm{~Gy}_{10}$ (26.1\% vs. 6.5\%). Another study by Kim et al. [11] also reported that the response of PVT was higher if the prescribed BED was higher than $58 \mathrm{~Gy}_{10}$ (54.6\% vs. 20\%). The results of those studies suggested that BEDs above $60 \mathrm{~Gy}_{10}$ might be related to better response rates.

In the current study, the prescribed dose was $40 \mathrm{~Gy} / 16$ fractions or $45 \mathrm{~Gy} / 25$ fractions, which was translated to the BEDs of $53.1 \mathrm{~Gy}_{10}$ and $50 \mathrm{~Gy}_{10}$, respectively. The response rate of the patients was 40\%, and the 1-year survival rate was $15 \%$.

The reason for these relatively low values of the response rate and 1-year survival rate might be a lower prescribed dose compared with the doses used in other published studies. And as tumor thrombus is considered unmeasurable lesion, the criteria for the response evaluation differs between studies. For example, Ishikura et al. [28] used ultrasonography for the evaluation of response. But Kim et al. [5], Kim et al. [11], Lee et al. [18], and Lin et al. [29] with other studies used CT to evaluate the response of therapy. Even if the studies used same method for evaluation of response, the definition of criteria for response differed between studies. These differences in evaluation of response could be biased.

Patients who were included in this study were treated with two types of prescribed doses: 45 Gy/25 fractions of a conventional fractionated schedule, and $40 \mathrm{~Gy} / 16$ fractions of a hypofractionated schedule with a larger fraction size. As the tumor size was relatively larger in the conventionally fractionated group than in the hypofractionated group $(100 \mathrm{~mL}$ and $38 \mathrm{~mL}$, respectively), the doses delivered to the surrounding 
normal tissues were relatively higher for the conventionally fractionated group. Although the doses delivered to the normal tissues differed, there was no significant difference in toxicity between the two groups. The response rate and the patients' survival demonstrated no significant difference between the two groups. Based on these data, both treatment options could be considered as comparable treatment options. As the PVT or HVT patients usually have dismal outcome and short life expectancy, rapid course treatment of hypofractionation could be a better strategy for these patients.

To escalate the prescribed dose, precise RT delivery is necessary for sparing the surrounding normal tissue. Because the liver is located just under the diaphragm, there is a relatively large organ movement owing to respiration. As in other studies, PTV of 1-2 cm margin from GTV was required for compensating daily setup variations and respiratory motion of the liver. This increased the dose to OAR, and increased the risk and severity of the RT-induced toxicity. With the development of more sophisticated RT techniques, such as tracking, gaiting, or abdominal compression, the organ movement can be minimized. The control of the organ movement could reduce PTV margins from GTV. By reducing these margins, a lower dose delivered to OAR and a higher dose delivered to a tumor can be expected.

Most of the acute complications were limited to grade 1 or 2 mild toxicities. They were general weakness, nausea, or abdominal pain, and were easily managed with conservative managements. Four patients had suffered grade 3 toxicity hematemesis which were discovered to be due to varix bleeding. By retrospectively reviewing those patients, we determined that all patients had varix before RT, confirmed by EGD performed before RT. The dose delivered to esophagus, stomach, and duodenum varied; thus, it was unlikely that variceal bleeding was caused by RT. Although hematemesis of patients included in this study was unlikely to have been caused by RT, attention should be given to doses delivered to esophagus, stomach, and duodenum.

RILD is one of the most common and important radiationinduced complications in the liver. With advances in RT techniques, the incidence and severity of RILD could be reduced with careful planning. It has been reported that RILD can be prevented if the mean dose to the liver is kept under $28 \mathrm{~Gy}$, or if the volume of the normal liver receiving more than 50 Gy $\left(V_{50}\right)$ is kept under 33\% $[5,11,20,23,30]$. Those liver tolerance dose guidelines adopted in previous studies were based on quantitative analyses of normal tissue effects in the clinic (OUANTEC) data and guideline suggested by Emami et al. [31]. When treating patients with therapeutic partial liver RT with standard fractionation for $5 \%$ or less risk of RILD, QUANTEC suggests that the mean dose to the liver should be under 28 Gy for primary liver cancer, and under $32 \mathrm{~Gy}$ for liver metastases [32]. Emami et al. [31] suggest that, for $5 \%$ or less risk of liver failure, $V_{50}$ should be under $33 \% ; V_{35}$, under $50 \%$; and $V_{30}$, under $100 \%$

In the current study, the normal liver dose constraints were as follows: $V_{25}$ was kept under $50 \%$, and the mean total dose to the liver was kept under $30 \mathrm{~Gy}$. By reviewing the dose volume histogram data of the patients in this study, normal tissue constraints on the liver were checked on the basis of the QUANTEC data and guidelines suggested by Emami et al. [31] For our 50 patients, the mean dose to the liver was $19.3 \mathrm{~Gy}$; the mean $V_{50}, 0 \%$; the mean $V_{35}$ of $50,19.6 \%$; and the mean $V_{30}, 25.4 \%$. All dose constraints suggested by the QUANTEC data and by Emami et al. [31] were achieved for all patients.

After radiotherapy, 1 patient met the criteria for non-classic RILD. The patient was a 57-year old man who was treated for right PVT with the $45 \mathrm{~Gy} / 25$ fractions schedule. The mean dose to the liver was $17.6 \mathrm{~Gy}_{\text {; }}$ the $\mathrm{V}_{50}, 0 \%$; the $\mathrm{V}_{35}, 20 \%$; the $\mathrm{V}_{30}$, $24 \%$; and the $V_{25}, 31 \%$. All dose constraints, suggested by our study, QUANTEC, and Emami et al. [31] were met. Three months after radiotherapy, his AST and ALT levels were 10,718 IU/L and 1,389 IU/L, respectively. The criteria for non-classic RILD is an elevation of the AST and ALT levels fivefold above the normal limit or level before RT; thus, this patient met the criteria for non-classic RILD. However, the patient had undergone TACE 5 days before the elevation of transaminases; thus, it was unclear whether the elevation of transaminases was induced by radiation or intervention. No patient met the criteria for classic RILD. Based on the current data on liver toxicity, although this was a retrospective study, dose limitation adopted for liver in this study could be considered safe.

Child-Pugh score reflects patients' current hepatic function, and increase in Child-Pugh score could reflect the deterioration of hepatic function. In current study, 25 patients (53\%) showed increase in Child-Pugh score after RT, and it was directly linked with the survival of patients (Tables 2 and 3). As all the patients included in this study were inoperable advanced HCC patients with tumor thrombus, it is hard to determine whether this change of Child-Pugh score was due to RT or natural course of HCC. But it might be important to note that if Child-Pugh score increases more than 2 points, it could be correlated with worse prognosis.

This study had several limitations. First, the small patient group size (47 patients) could have masked the difference 
between the prognostic factors determining the patients' survival. Prognostic significance of these factors could be made clearer by considering larger groups of patients. Second, because this study was a retrospective one, the population was not controlled and could be biased. Third, there were patients who were lost during follow-up, and patients who are still alive. These patients could have negative effects to the result of current study, so longer follow-up of these patients might be needed.

In conclusion, conventional radiotherapy for PVT or HVT could be a reasonable palliative treatment option without incurring severe toxicity. The prognosis of patient was affected by following factors; changes in Child-Pugh score after RT, and response to $\mathrm{RT}$, revealed by a multivariate analysis.

The prescribed dose in this study was relatively low compared with that in other studies, and the treatment results were also relatively insignificant (response rate of $40 \%$, and 1 year overall survival of 15\%). It might be necessary to consider escalating the prescribed dose for obtaining better outcomes.

\section{Conflict of Interest}

No potential conflict of interest relevant to this article was reported.

\section{References}

1. El-Serag HB, Rudolph KL. Hepatocellular carcinoma: epidemiology and molecular carcinogenesis. Gastroenterology 2007;132:2557-76.

2. Stuart KE, Anand AJ, Jenkins RL. Hepatocellular carcinoma in the United States: prognostic features, treatment outcome, and survival. Cancer 1996;77:2217-22.

3. Pirisi $M_{1}$ Avellini $C_{\text {, Fabris }}$, et al. Portal vein thrombosis in hepatocellular carcinoma: age and sex distribution in an autopsy study. J Cancer Res Clin Oncol 1998;124:397-400.

4. Fong $Y$, Sun $R L$, Jarnagin $W$, Blumgart $L H$. An analysis of 412 cases of hepatocellular carcinoma at a Western center. Ann Surg 1999;229:790-9.

5. Kim DY, Park W, Lim DH, et al. Three-dimensional conformal radiotherapy for portal vein thrombosis of hepatocellular carcinoma. Cancer 2005;103:2419-26.

6. Minagawa M, Makuuchi M, Takayama T, Ohtomo K. Selection criteria for hepatectomy in patients with hepatocellular carcinoma and portal vein tumor thrombus. Ann Surg 2001;233:379-84.

7. Ando $E_{1}$ Tanaka $M$, Yamashita $F$, et al. Hepatic arterial infusion chemotherapy for advanced hepatocellular carcinoma with portal vein tumor thrombosis: analysis of 48 cases. Cancer 2002;95:588-95.

8. Kondo K, Chijiiwa K, Kai M, et al. Surgical strategy for hepatocellular carcinoma patients with portal vein tumor thrombus based on prognostic factors. J Gastrointest Surg 2009;13:1078-83.

9. Lee DS, Seong J. Radiotherapeutic options for hepatocellular carcinoma with portal vein tumor thrombosis. Liver Cancer 2014;3:18-30.

10. Llovet JM, Bustamante J, Castells $A$, et al. Natural history of untreated nonsurgical hepatocellular carcinoma: rationale for the design and evaluation of therapeutic trials. Hepatology 1999;29:62-7.

11. Kim TH, Kim DY, Park JW, et al. Three-dimensional conformal radiotherapy of unresectable hepatocellular carcinoma patients for whom transcatheter arterial chemoembolization was ineffective or unsuitable. Am J Clin Oncol 2006;29:56875.

12. Zeng ZC, Fan J, Tang ZY, et al. Prognostic factors for patients with hepatocellular carcinoma with macroscopic portal vein or inferior vena cava tumor thrombi receiving external-beam radiation therapy. Cancer Sci 2008;99:2510-7.

13. Huang YJ, Hsu HC, Wang $C Y$, et al. The treatment responses in cases of radiation therapy to portal vein thrombosis in advanced hepatocellular carcinoma. Int J Radiat Oncol Biol Phys 2009;73:1155-63.

14. Kim SH, Kang MK, Yea JW, Kim SK, Choi JH, Oh SA. The impact of beam angle configuration of intensity-modulated radiotherapy in the hepatocellular carcinoma. Radiat Oncol J 2012;30:146-51.

15. Zhang XB, Wang JH, Yan ZP, Qian S, Du SS, Zeng ZC. Hepatocellular carcinoma with main portal vein tumor thrombus: treatment with 3-dimensional conformal radiotherapy after portal vein stenting and transarterial chemoembolization. Cancer 2009;115:1245-52.

16. Rim CH, Yang DS, Park YJ, Yoon WS, Lee JA, Kim CY. Effectiveness of high-dose three-dimensional conformal radiotherapy in hepatocellular carcinoma with portal vein thrombosis. Jpn J Clin Oncol 2012;42:721-9.

17. Yoon SM, Lim YS, Won HJ, et al. Radiotherapy plus transarterial chemoembolization for hepatocellular carcinoma invading the portal vein: long-term patient outcomes. Int $J$ Radiat Oncol Biol Phys 2012;82:2004-11.

18. Lee JH, Kim DH, Ki YK, et al. Three-dimensional conformal radiotherapy for portal vein tumor thrombosis alone in advanced hepatocellular carcinoma. Radiat Oncol J 2014;32:170-8.

19. Bruix J, Sherman M; American Association for the Study of Liver Diseases. Management of hepatocellular carcinoma: an update. Hepatology 2011;53:1020-2. 
20. Cheng JC, Wu JK, Huang CM, et al. Radiation-induced liver disease after three-dimensional conformal radiotherapy for patients with hepatocellular carcinoma: dosimetric analysis and implication. Int J Radiat Oncol Biol Phys 2002;54:156-62.

21. Cheng JC, Wu JK, Huang $C M$, et al. Radiation-induced liver disease after radiotherapy for hepatocellular carcinoma: clinical manifestation and dosimetric description. Radiother Oncol 2002;63:41-5.

22. Dawson LA, Normolle D, Balter JM, McGinn CJ, Lawrence TS, Ten Haken RK. Analysis of radiation-induced liver disease using the Lyman NTCP model. Int J Radiat Oncol Biol Phys 2002;53:810-21.

23. Liang $S X, Z h u X D, X u Z Y$, et al. Radiation-induced liver disease in three-dimensional conformal radiation therapy for primary liver carcinoma: the risk factors and hepatic radiation tolerance. Int J Radiat Oncol Biol Phys 2006;65:426-34.

24. Son SH, Jang HS, Jo IY, et al. Significance of an increase in the Child-Pugh score after radiotherapy in patients with unresectable hepatocellular carcinoma. Radiat Oncol 2014:9:101.

25. Tazawa J, Maeda M, Sakai Y, et al. Radiation therapy in combination with transcatheter arterial chemoembolization for hepatocellular carcinoma with extensive portal vein involvement. J Gastroenterol Hepatol 2001;16:660-5.
26. Yamada $K_{1}$ Izaki $K_{1}$ Sugimoto $K_{1}$ et al. Prospective trial of combined transcatheter arterial chemoembolization and three-dimensional conformal radiotherapy for portal vein tumor thrombus in patients with unresectable hepatocellular carcinoma. Int J Radiat Oncol Biol Phys 2003;57:113-9.

27. Toya $R$, Murakami $R, B a b a ~ Y$, et al. Conformal radiation therapy for portal vein tumor thrombosis of hepatocellular carcinoma. Radiother Oncol 2007;84:266-71.

28. Ishikura S, Ogino T, Furuse J, et al. Radiotherapy after transcatheter arterial chemoembolization for patients with hepatocellular carcinoma and portal vein tumor thrombus. Am J Clin Oncol 2002;25:189-93.

29. Lin CS, Jen YM, Chiu SY, et al. Treatment of portal vein tumor thrombosis of hepatoma patients with either stereotactic radiotherapy or three-dimensional conformal radiotherapy. Jpn J Clin Oncol 2006;36:212-7.

30. Guha C, Kavanagh BD. Hepatic radiation toxicity: avoidance and amelioration. Semin Radiat Oncol 2011;21:256-63.

31. Emami B, Lyman J, Brown A, et al. Tolerance of normal tissue to therapeutic irradiation. Int J Radiat Oncol Biol Phys 1991;21:109-22.

32. Pan CC, Kavanagh BD, Dawson LA, et al. Radiationassociated liver injury. Int J Radiat Oncol Biol Phys 2010;76(3 Suppl):S94-100. 